S. Kwok, M. Dopita, and R. Sutherland, eds.

\title{
HST Observations of Young Planetary Nebulae
}

\author{
Sun Kwok and Kate Y.L. Su \\ Dept. of Phys. and Astr., U. of Calgary, Calgary, Canada T2N 1N4 \\ Raghvendra Sahai \\ Jet Propulsion Laboratory, Pasadena, CA 91109, U.S.A.
}

\section{Observations, results and discussion}

Seven compact $\mathrm{PNe}$ with high radio surface brightness (and therefore presumably young) were observed with the $H S T$ WFPC2 under cycle 8 program 8307 (PI: S. Kwok). Observations were made with three narrow-band filters, F656N $(\mathrm{H} \alpha), \mathrm{F} 658 \mathrm{~N}([\mathrm{NII}])$ and $\mathrm{F} 502 \mathrm{~N}$ ([OIII]). The [NII] images of six of the nebulae are shown in Figure 1.

All PNe show one or more inner shells accompanied by one or more pairs of bipolar lobes. He2-447, IC 5117 and M3-35 show a bright inner shell with two pairs of bipolar lobes oriented at different angles. NGC 6790 has two inner shells of similar orientations. M1-61 has at least two inner shells and at least one, probably two, pairs of bipolar lobes. The most interesting structure is seen in NGC 6881 where several rings can be seen both in the torus and in the lobes. The plane of these rings are perpendicular to the major axis of the lobes and resemble the rings seen in the $H S T$ image of SN $1987 \mathrm{~A}$. These rings represent a different phenomenon to the concentric arcs seen in proto-PNe and PNe, which are spherical shells projected onto the plane of the sky (Kwok et al. 2001).

The multi-polar lobes observed resemble the point-symmetric structures seen in large nebulae such as KjPn8 (López et al. 1995) and NGC 2440 (Miranda et al. 1999). Similar structures have also been previously reported in the young PNe He2-47 and M1-37 (Sahai 2000). The existence of more than one polar axis suggests that simultaneous collimated outflows in more than one direction, or the outflow direction has changed with time (sometimes referred to as bipolar, rotating, episodic jets, BRET). The approximately equal sizes of the lobes seems to favor the first hypothesis. These results suggest that the fast outflows responsible for the shaping of PNe are not spherically symmetric, but are highly collimated.

\section{References}

Kwok, S., Su, K.Y.L., \& Stoesz, J.A. 2001, in Post-AGB Stars as a Phase of Stellar Evolution, eds. R. Szczerba \& S.K. Gorny, (Kluwer), p. 115

López, J. A., Vazquez, R., and Rodriguez, L. F. 1995, ApJ, 455, L63

Miranda et al. 1999, ApJ, 117, 1421

Sahai, R. 2000, ApJ, 537, L43 

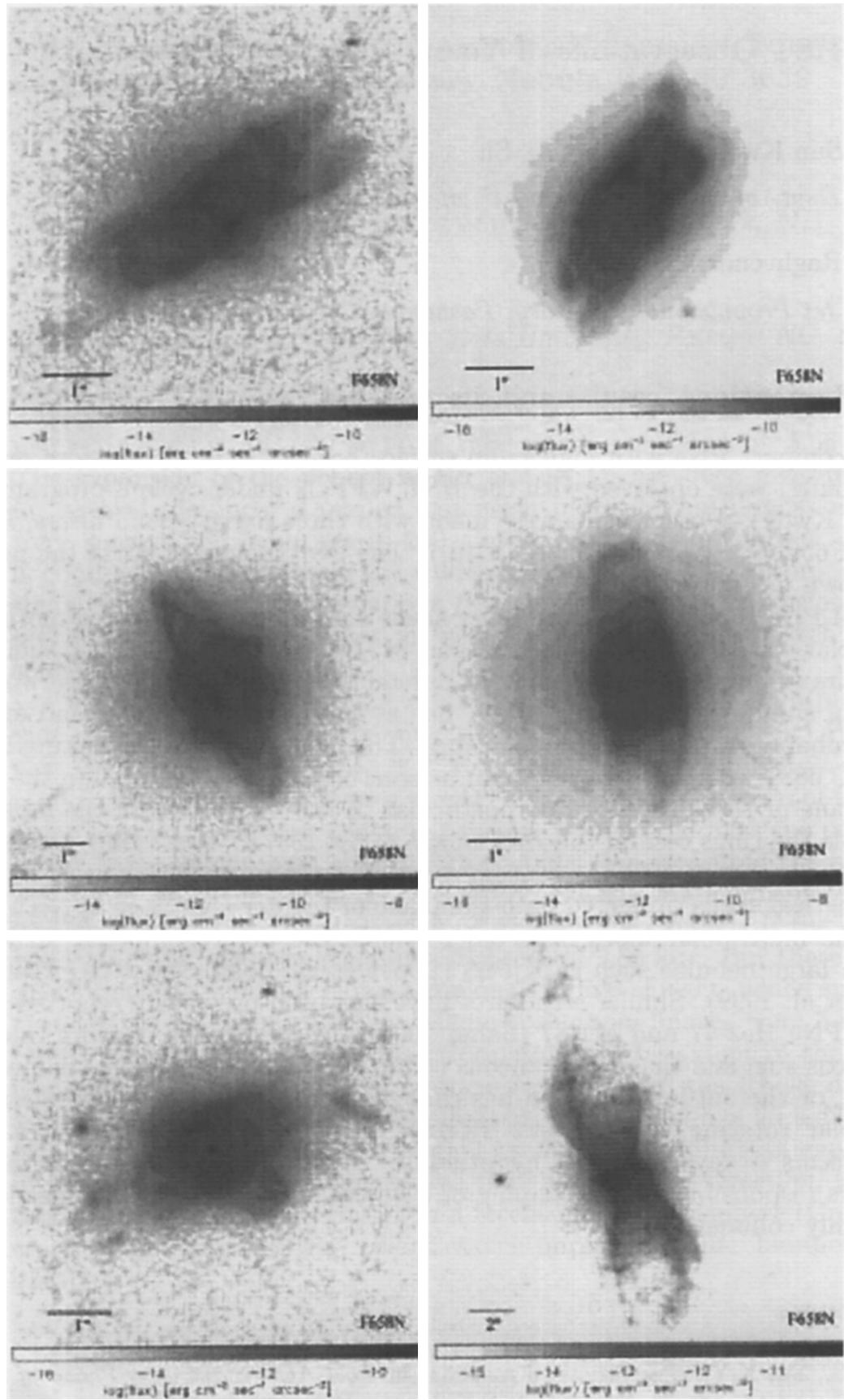

Figure 1. HST WFPC2 [NII] images of He2-447 (top left), IC 5117 (top right), M1-61 (middle left), NGC 6790 (middle right), M3-35 (bottom left), and NGC 6881 (bottom right). 